\title{
NOTE ON COMPACT SETS OF FIRST BAIRE CLASS FUNCTIONS
}

\author{
ROMAN POL ${ }^{1}$
}

\begin{abstract}
We give an example of a separable compact subspace of the space of first Baire class functions on the irrationals which cannot be embedded in the space of first Baire class functions on the Cantor set, both of the function spaces being considered with the pointwise topology. This answers a question asked in [Ta, Problem 14-2-4].
\end{abstract}

1. Introduction. Our terminology follows Kuratowski's Topology [Ku]; in particular, a function is of first Baire class if it is a pointwise limit of a sequence of continuous functions and a set is analytic if it is a continuous image of the irrationals. Given a metrizable separable space $X$ we denote by $B_{1}(X)$ the space of real-valued first Baire class functions on $X$ endowed with the pointwise topology.

In this note we give an example of a separable compact space $K$ which can be embedded in $B_{1}\left(\omega^{\omega}\right)$, $\omega^{\omega}$ being the irrationals, but cannot be embedded in $B_{1}\left(2^{\omega}\right)$, where $2^{\omega}$ is the Cantor set (and hence $K$ cannot be embedded in any $B_{1}(X)$ with a compact metrizable $X$, any such $X$ being a continuous image of $2^{\omega}$ ).

This answers a question asked by M. Talagrand [Ta, Problem 14-2-4].

Our reasoning is based on an existence of an analytic but non-Borel set in the unit interval and on the classical Kunugui-Novikov Theorem which states that the projection of a Borel set in the plane whose vertical sections are $\sigma$-compact is a Borel set.

2. A compact subspace $K$ of $B_{1}\left(\omega^{\omega}\right)$. Actually, we shall define a compact subset $K$ of a space $B_{1}(S)$ for some analytic set $S$, but then, if $u: \omega^{\omega} \rightarrow S$ is a continuous map of the irrationals onto $S$, the adjoint map $f \rightarrow f \circ u$ embeds $K$ into $B_{1}\left(\omega^{\omega}\right)$.

Let $I=[0,1]$ be the unit interval and let $\mathbf{T}$ be the collection of all subintervals of the form $[m / n, m+1 / n]$, where $m$ and $n$ are natural and $0 \leqslant m \leqslant n-1$.

Let $S$ be an analytic but non-Borel subset of $I$, dense in $I$ and disjoint from rational numbers.

For each $T \in$ T let $f_{T}: S \rightarrow\{0,1\}$ be 1 on $T \cap S$ and 0 on $S \backslash T$, for each $x \in S$ let $f_{x}: S \rightarrow\{0,1\}$ take the value 1 at $x$ and 0 otherwise, and finally, let $f_{\varnothing}$ be the function on $S$ constantly equal to 0 .

Received by the editors December 18, 1984.

1980 Mathematics Subject Classification. Primary 54H05; Secondary 04A15.

${ }^{1}$ This paper was written while the author was visiting the Auburn University, Alabama. The author gratefully acknowledges the hospitality of the Auburn University.

(C) 1986 American Mathematical Society $0002-9939 / 86 \$ 1.00+\$ .25$ per page 
The subspace of $B_{1}(S)$ we shall consider is

$$
K=\left\{f_{\varnothing}\right\} \cup\left\{f_{x}: x \in S\right\} \cup\left\{f_{T}: T \in \mathbf{T}\right\} \subset B_{1}(S) .
$$

The topological structure of $K$ is quite simple: points $f_{T}$ are isolated in $K$ (and form a dense set in $K$ ), basic neighborhoods of a point $f_{x}$ are of the form $\left\{f_{x}\right\} \cup\left(\left\{f_{T}: x \in T\right\} \backslash F\right)$, where $F$ is a finite set, and $f_{\varnothing}$ is a point at infinity of the locally compact space $\left\{f_{T}: T \in \mathbf{T}\right\} \cup\left\{f_{x}: x \in S\right\}$.

3. The space $K$ cannot be embedded in $B_{1}\left(2^{\omega}\right)$. Assume on the contrary that there exists a homeomorphic embedding $H: K \rightarrow B_{1}\left(2^{\omega}\right)$.

Let us associate with $H$ a subset of the product $I \times 2^{\omega}$ defined by

$$
E=\left\{(x, y) \in S \times 2^{\omega}: H\left(f_{x}\right)(y) \neq H\left(f_{\varnothing}\right)(y)\right\} .
$$

Since $f_{x}$ and $f_{\varnothing}$ are both of first Baire class, for each $x \in S$ the set $\{y$ : $\left.H\left(f_{x}\right)(y) \neq H\left(f_{\varnothing}\right)(y)\right\}$ is an $F_{\sigma}$ set in $2^{\omega}$, and hence

$$
\text { each vertical section of } E \text { is } \sigma \text {-compact. }
$$

Let us show that

$$
E \text { is a Borel set in } I \times 2^{\omega} \text {. }
$$

It is enough to verify that $E$ can also be described by the following formula, where $m, k, n$ are natural numbers and $\mathbf{T}_{n}=\{T \in \mathbf{T}$ : diameter $T \leqslant 1 / n\}$ :

$$
E=\bigcup_{m} \bigcap_{k} \bigcup_{n>k} \bigcup_{T \in \mathbf{T}_{n}} T \times\left\{y:\left|H\left(f_{T}\right)(y)-H\left(f_{\varnothing}\right)(y)\right| \geqslant 1 / m\right\} .
$$

If $(x, y) \in E$, i.e. $x \in S$ and $H\left(f_{x}\right)(y) \neq H\left(f_{\varnothing}\right)(y)$, then by the continuity of $H$ with respect to the pointwise topology, the inequality $H\left(f_{T}\right)(y) \neq H\left(f_{\varnothing}\right)(y)$ holds for all but finitely many intervals $T$ containing $x$ (cf. a description of $K$ at the ending of \$2), and since the diameters of the intervals in $\mathbf{T}$ converge to zero, it follows that $(x, y)$ belongs to the set on the right-hand side of formula (4).

On the other hand, let us assume that $(x, y)$ belongs to the set on the right-hand side of (4). Then there exist an $m$ and an infinite set of intervals $\mathbf{L} \subset \mathbf{T}$ such that for each $T \in \mathbf{L}, x \in T$ and $\left|H\left(f_{T}\right)(y)-H\left(f_{\varnothing}\right)(y)\right| \geqslant 1 / m$.

By the continuity of $H$, there exists a neighborhood $V$ of the point $f_{\varnothing}$ in $K$ such that for all $f$ in $V$ the inequality $\left|H(f)(y)-H\left(f_{\varnothing}\right)(y)\right|<1 / m$ holds; one can assume that $V$ is the trace on $K$ of a basic neighborhood of $f_{\varnothing}$ in $B_{1}(S)$, i.e. that for some finite set $F \subset S, V=\{f \in K: f(s)=0$ for all $s \in F\}$. For each $T \in \mathbf{L}, f_{T}$ does not belong to $V$, so $T$ contains a point from $F$ and the set $F$ being finite, there exists a point in $F$ which belongs to infinitely many intervals $T_{1}, T_{2}, \ldots$ from $\mathbf{L}$.

The diameters of $T_{i}$ converge to zero, and since both $x$ and $s$ belong to each $T_{i}$, it follows that $x=s$, i.e. $x \in S$. Moreover, $f_{x}=\lim _{i} f_{T_{i}}$ and hence by the continuity of $H,\left|H\left(f_{x}\right)(y)-H\left(f_{\varnothing}\right)(y)\right| \geqslant 1 / m$, i.e. $(x, y) \in E$. 
We are now in position to derive a contradiction from an existence of $H$ : by the classical Kunugui-Novikov Theorem [K-M], the projection of the Borel set $E$ with $\sigma$-compact vertical sections onto the $I$-axis should be a Borel set, but, $H$ being injective, $H\left(f_{x}\right) \neq H\left(f_{\varnothing}\right)$ for each $x \in S$, so this projection must be equal to $S$ (see (1)) which has been chosen non-Borel.

\section{REFERENCES}

[Ku] K. Kuratowski, Topology, vol. I, PWN, Warszawa, 1966.

[K-M] K. Kuratowski and A. Mostowski, Set theory with an introduction to descriptive set theory, PWN, Warszawa, 1976.

[Ta] M. Talagrand, Pettis integral and measure theory, Mem. Amer. Math. Soc. No. 51 (1984).

Department of Mathematics, U. W., PKin 1XP., 00 - 901 WarszaWa, Poland 